\title{
Asymmetric strain relaxation in patterned SiGe layers: A means to enhance carrier mobilities in Si cap layers
}

D. Buca, B. Holländer, S. Feste, St. Lenk, H. Trinkaus, S. Mantl, R. Loo, and M. Caymax

Citation: Appl. Phys. Lett. 90, 032108 (2007); doi: 10.1063/1.2431702

View online: https://doi.org/10.1063/1.2431702

View Table of Contents: http://aip.scitation.org/toc/apl/90/3

Published by the American Institute of Physics

\section{Articles you may be interested in}

Anisotropy of strain relaxation in (100) and (110) Si/SiGe heterostructures Journal of Applied Physics 111, 014904 (2012); 10.1063/1.3672447

Short-circuit photocurrent in epitaxial lead zirconate-titanate thin films Journal of Applied Physics 101, 064109 (2007); 10.1063/1.2560217

Strained Ge channel p-type metal-oxide-semiconductor field-effect transistors grown on $\mathrm{Si}_{1-\mathrm{x}} \mathrm{Ge}_{\chi} / \mathrm{Si}$ virtual substrates

Applied Physics Letters 79, 3344 (2001); 10.1063/1.1417515

Controlling threading dislocation densities in Ge on Si using graded SiGe layers and chemical-mechanical polishing

Applied Physics Letters 72, 1718 (1998); 10.1063/1.121162

Effect of asymmetric strain relaxation on dislocation relaxation processes in heteroepitaxial semiconductors Journal of Applied Physics 121, 075302 (2017); 10.1063/1.4975789

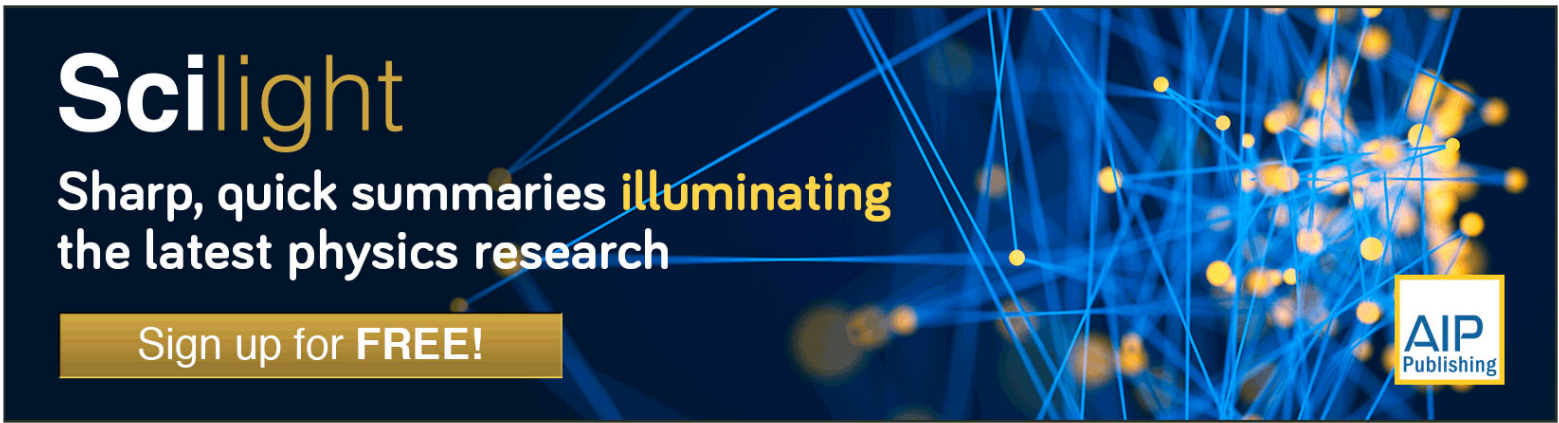




\title{
Asymmetric strain relaxation in patterned SiGe layers: A means to enhance carrier mobilities in Si cap layers
}

\author{
D. Buca, ${ }^{\text {a) }}$ B. Holländer, S. Feste, St. Lenk, H. Trinkaus, and S. Mantl \\ Institute of Bio- and Nanosystems, Forschungszentrum Jülich GmbH, D-52425 Jülich, \\ Germany Center of Nanoelectronic Systems for Information Technology (CNI), \\ Forschungszentrum Jülich GmbH, D-52425 Jülich, Germany \\ R. Loo and M. Caymax \\ IMEC, Kapeldreef 75, B-3001 Leuven, Belgium
}

(Received 21 August 2006; accepted 6 October 2006; published online 18 January 2007)

\begin{abstract}
Strain relaxation in patterned $\mathrm{Si}_{0.77} \mathrm{Ge}_{0.23}$ stripes grown on $\mathrm{Si}(001)$ by chemical vapour deposition was investigated after $\mathrm{He}^{+}$ion implantation and annealing. Ion channeling measurements indicate asymmetric strain relaxation with a significantly higher residual strain parallel to the stripes than perpendicular to the stripes. These results are confirmed by plan view transmission electron microscopy showing a much higher density of misfit dislocations running along the stripes than across the stripes. Estimates based on a piezoresistivity model indicate significant enhancements of electron and hole mobilities for asymmetrically strained Si cap layers on such SiGe stripes. (C) 2007 American Institute of Physics. [DOI: 10.1063/1.2431702]
\end{abstract}

Application of stress is an efficient method to enhance carrier mobilities in semiconductors. Epitaxially grown $\mathrm{Si}$ on a relaxed SiGe buffer layer shows symmetric (twodimensionally isotropic) biaxial stress and a substantially larger electron mobility ${ }^{1}$ which increases with increasing stress. On the other hand, stressor layers on transistors produce uniaxial stress and enhance the hole mobility. ${ }^{2}$ When biaxially stressed silicon on $\mathrm{SiO}_{2}$ is patterned into small structures, strain relaxation preferentially along the shorter dimensions is observed. ${ }^{3}$ Asymmetric stress relaxation via plastic flow was also observed for patterned $\mathrm{Si} / \mathrm{SiGe}$ stripes on viscoelastic glass. ${ }^{4}$ In this letter we will present a method to fabricate strongly asymmetrically strained silicon with improved mobilities for electrons and holes.

We have recently shown that in thin $\mathrm{Si}$ layers on $\mathrm{SiGe} / \mathrm{Si}$ heterostructures, high biaxial tensile stresses can be generated by "strain transfer" from the relaxing SiGe layer to the Si layer. ${ }^{5}$ The efficiency of this process was studied as a function of the Si layer thickness. The strain induced in the $\mathrm{Si}$ layer upon relaxation of the SiGe layer was described by

$$
\varepsilon_{\mathrm{Si}}=\eta R x f_{\mathrm{Ge}, \mathrm{Si}} \text {. }
$$

Here $f_{\mathrm{Ge}, \mathrm{Si}}=4.2 \%$ is the lattice mismatch between $\mathrm{Ge}$ and $\mathrm{Si}, R$ is the degree of relaxation of the $\mathrm{Si}_{1-x} \mathrm{Ge}_{x}$ epilayer, and $\eta$ is the "strain transfer efficiency" which was shown to reach 100\% for Si layer thicknesses below about $10 \mathrm{~nm}$.

A key step in this strain transfer process is strain relaxation in the SiGe epilayers. We have recently shown that this can be efficiently achieved by $\mathrm{He}^{+}$ion implantation and subsequent thermal annealing. ${ }^{5,6}$ In a model of this process, ${ }^{6}$ we have assumed that the narrow defect band formed upon ion implantation underneath the $\mathrm{SiGe} / \mathrm{Si}$-substrate interface provides a high density of dislocation loops during annealing, part of which glides to the interface and evolves from there into dislocation arms consisting of segments of misfit dislocations (MDs) in the interface and threading dislocations (TDs) through the strained SiGe layer. The stress driven

\footnotetext{
${ }^{a)}$ Electronic mail: d.m.buca@fz-juelich.de
}

propagation of the TD segments through the $\mathrm{SiGe}$ layer along the [110] and $[-110]$ directions is associated with an extension of the corresponding MD segments involving an increasing strain relaxation. Interaction and mutual annihilation of TD segments result in a reduction of the TD density to an acceptably low level. ${ }^{7}$ In the final state, a long MD consists of the trails of many TDs. Accordingly, the final MD density and the corresponding level of strain relaxation are determined by the density of the loops contributing as dislocation sources and the average path length of the TDs before they annihilate or stop.

Since the probabilities for both the generation of loops with different but crystallographically equivalent Burgers vectors and the average path lengths of the resulting dislocations extending in the two in-plane $\langle 110\rangle$ directions are equal, the relaxation of the $\mathrm{SiGe}$ layer is symmetric (isotropic), i.e., the $\mathrm{SiGe}$ crystal structure remains tetragonal during relaxation. Our model suggests that this symmetry can be broken by patterning the SiGe layer into micrometer narrow [110] stripes for which the average path length of TDs moving in the $[-110]$ direction would be shortened by the two stripe boundaries, resulting in a reduction of the MD density in this direction and a corresponding reduction of the degree of relaxation in the [110] direction. This idea is illustrated in Fig. 1.

In the present letter, we report on a study of asymmetric stress relaxation in patterned $\mathrm{SiGe}$ stripe structures on $\mathrm{Si}(001)$ wafers. Epitaxial $\mathrm{Si} / \mathrm{Si}_{0.77} \mathrm{Ge}_{0.23}(6 \mathrm{~nm} / 180 \mathrm{~nm})$ heterostructures were grown on $\mathrm{Si}(001)$ by chemical vapor deposition in an ASM Epsilon ${ }^{\circledR}$ production tool. The samples were implanted with $7 \times 10^{15} \mathrm{He}$ ions $/ \mathrm{cm}^{2}$ at an energy of $45 \mathrm{keV}$. Stripes along the [110] direction with widths varying between 0.8 and $10 \mu \mathrm{m}$ were patterned using standard optical lithography and etching. Finally, the structures were annealed at $850{ }^{\circ} \mathrm{C}$ for $10 \mathrm{~min}$ in nitrogen to achieve strain relaxation.

The measurement of the strains in the stripe structures turned out to be difficult. Raman spectroscopy provides only an average strain and $\mathrm{x}$-ray diffraction has been shown to 


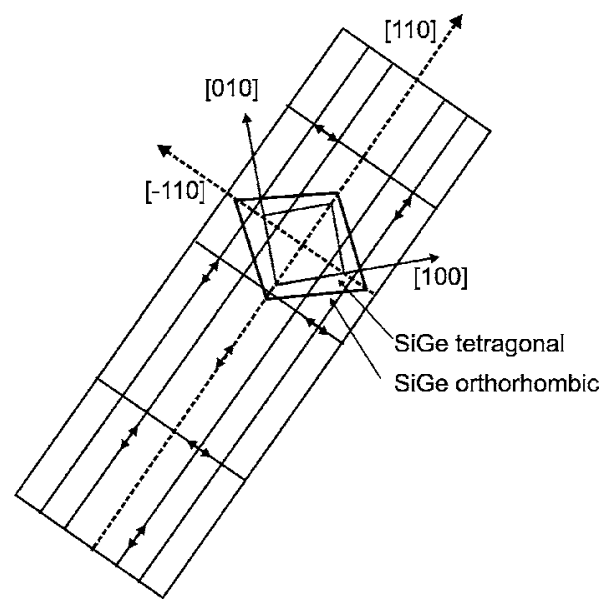

FIG. 1. Illustration of a misfit network after asymmetric strain relaxation in a SiGe stripe on (001) Si. The limitation of the paths of TDs in the $[-110]$ direction by the stripe boundary results in a reduced MD density in this compared to the [110] direction and a correspondingly reduced degree of relaxation in the [110] direction. By this, the square basis of the tetragonal unit cell of the SiGe crystal is distorted to a rhombic basis of an orthorhombic unit cell in the $[110] /[-110] /[001]$ coordinate system.

underestimate strain contributions of low MD densities and to overestimate by this the asymmetry. ${ }^{8}$ In this work, we employ $\mathrm{He}$ ion channeling to measure strains. In detail, we perform ion channeling angular yield measurements of the Ge backscattering signal with a high-precision goniometer using $1.4 \mathrm{MeV} \mathrm{He}{ }^{+}$ions at a scattering angle of $170^{\circ}$.

Ion channeling angular yield scans provide absolute angles between various crystal directions and by this the lattice strains. ${ }^{9}$ For cubic lattices as unstrained Si or fully relaxed SiGe, the angle between [001] and [111], for instance, has a value of $54.736^{\circ}$. Compressive tetragonal strains in $\mathrm{SiGe} / \mathrm{Si}$ lead to smaller angles $\left(54.736^{\circ}-\Delta \theta\right)$ with $\Delta \theta$ $<\Delta \theta_{\mathrm{ps}}$ where $\Delta \theta_{\mathrm{ps}}$ denotes the maximum angular shift realized by the unrelaxed symmetrically stressed, pseudomorphic SiGe layer. For symmetric (isotropic) biaxial relaxation, the degree of relaxation may be defined as $R=1$ $-\left(\Delta \theta / \Delta \theta_{\mathrm{ps}}\right)$. We may directly extend this definition to asymmetrically relaxing [110] stripes if we assume the strain tensor to have the (110) and $(-110)$ mirror symmetries of the stripes (as expected for two sets of MDs along [110] and $[1-10]$, respectively). Then two different relaxation degrees, $R_{\|}$and $R_{\perp}$, may be defined by the two different changes, $\Delta \theta_{1,2}$ of the angles shifts between [001] and [111], and [001] and [-111], respectively: $R_{\|, \perp}=1-\Delta \theta_{1,2} / \Delta \theta_{\mathrm{ps}}$.

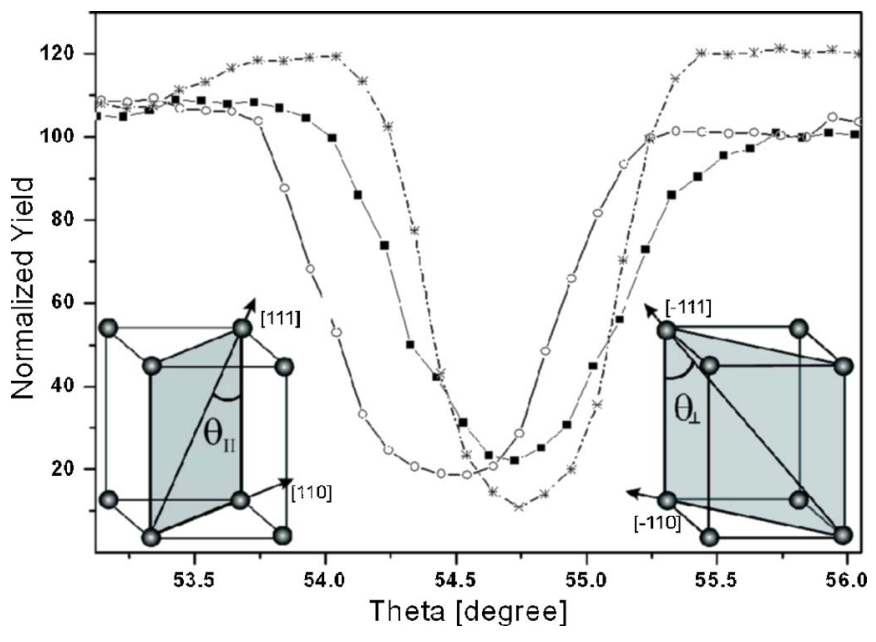

FIG. 2. (Color online) Channeling angular yield scans of the Ge backscattering signal from $0.8 \mu \mathrm{m}$ stripes measured across (empty circles) and along the stripe (full squares), respectively. The corresponding scan of a cubic $\mathrm{Si}$ crystal with its midpoint at $54.74^{\circ}$ is shown as reference (star symbols). The insets show the crystallographic directions of the two nonequivalent diagonals with the angles $\theta_{\|}$and $\theta_{\perp}$ along the stripe and parallel to the stripes, respectively.

Accordingly, for asymmetrically relaxing [110] stripes, we performed angular scans along the $(-110)$ plane through the [001] and the inclined [111] directions (along the patterned stripe, see Fig. 1) and along the (110) plane through the [001] and [-111] directions (across the patterned stripe). Figure 2 shows channeling angular yield scans of the Ge backscattering signal from a sample with a stripe width of $0.8 \mu \mathrm{m}$, both across and along the stripe. A corresponding scan for a cubic Si crystal is shown for reference. The midpoint of each scan represents the absolute angle between the [001] sample normal and the [-111] or the [111] directions, respectively (nonequivalent diagonals). The shift of the midpoint position provides direct evidence for the change of the tetragonal lattice to an orthorhombic lattice structure in the [110], [-110], [001] coordinate system.

The relaxation degrees of the SiGe stripes along, $R_{\|}$, and perpendicular, $R_{\perp}$, to the stripes are summarized in Table I. Obviously, the strain relaxation depends strongly on the width of the stripes. The very narrow stripes of $0.8 \mu \mathrm{m}$ in width show nearly full relaxation (95\%) perpendicular to the stripe direction but only small relaxation of about $34 \%$ along the stripes. The ratio of the two relaxation degrees, $R_{\perp} / R_{\|}$, has a value of approximately 2.8 . For comparison we have

TABLE I. Relaxation degrees, $R_{\|, \perp}$, along and perpendicular to SiGe stripes of different widths, together with strains $\varepsilon_{\|, \perp}$ and stresses $\sigma_{\|, \perp}$ as well as resistivity changes, $\Delta_{\|, \perp}$, expected to occur in thin Si cap on the SiGe stripes. $\Delta_{\|, \perp}$ values are calculated on the basis of piezoelectric measurements on Si (Ref. 10).

\begin{tabular}{|c|c|c|c|c|c|c|c|c|}
\hline \multicolumn{3}{|c|}{ SiGe stripes } & \multicolumn{6}{|c|}{ Virtual Si top layers } \\
\hline \multirow{3}{*}{$\begin{array}{l}\text { Linewidth } \\
\quad(\mu \mathrm{m})\end{array}$} & \multirow{2}{*}{\multicolumn{2}{|c|}{$\begin{array}{c}\text { Relaxation degree } \\
(\%)\end{array}$}} & \multirow{2}{*}{\multicolumn{2}{|c|}{ Strain $(\%) /$ stress $(\mathrm{GPa})$}} & \multicolumn{4}{|c|}{ Rel. res. change (\%) } \\
\hline & & & & & \multicolumn{2}{|c|}{$n-\mathrm{Si}$} & \multicolumn{2}{|c|}{$p$-Si } \\
\hline & $R_{\|}$ & $R_{\perp}$ & $\varepsilon_{\|} / \sigma_{\|}$ & $\varepsilon_{\perp} / \sigma_{\perp}$ & $\Delta_{\|}$ & $\Delta_{\perp}$ & $\Delta_{\|}$ & $\Delta_{\perp}$ \\
\hline$\rightarrow \infty$ & 70 & 70 & $0.68 / 1.22$ & $0.68 / 1.22$ & -59 & -59 & 6.7 & 6.7 \\
\hline 3.2 & 41 & 75 & $0.40 / 0.75$ & $0.72 / 1.27$ & -46 & -53 & -31 & 43 \\
\hline 2.8 & 35 & 77 & $0.34 / 0.65$ & $0.74 / 1.30$ & -43 & -52 & -39 & 50 \\
\hline 1.4 & 45 & 95 & $0.43 / 0.83$ & $0.92 / 1.60$ & -54 & -63 & -46 & 60 \\
\hline 0.8 & 34 & 95 & $0.33 / 0.65$ & $0.92 / 1.60$ & -48 & -61 & -59 & 71 \\
\hline
\end{tabular}




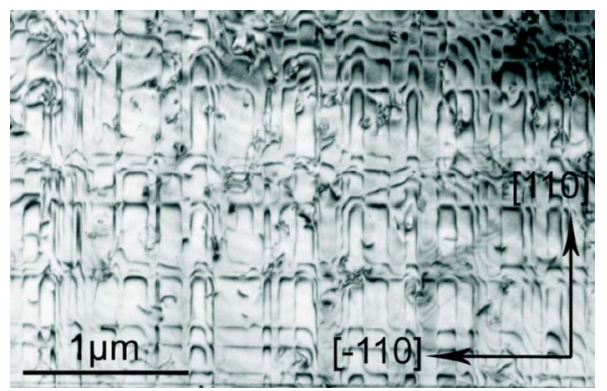

FIG. 3. (Color online) Plan view TEM micrograph of an asymmetric MD array in a $4 \mu \mathrm{m}$ wide stripe, where the misfit dislocation density perpendicular to the direction of the stripes is about a factor of 2.4 larger than that parallel to the stripes.

included in Table I data for symmetrically relaxed blanket SiGe layers obtained previously. ${ }^{5}$

According to the physical picture described above, this asymmetry in strain relaxation is due to the different path lengths of the TDs propagating along the two in-plane $\langle 110\rangle$ directions. If the TD path in the [-110] direction is limited by the stripe boundary, the density of MDs in this direction is reduced as illustrated by the thin line pattern in Fig. 1. This observation is also confirmed by plan view transmission electron microscopy (PV-TEM). Figure 3 shows a PV-TEM micrograph of a $4 \mu \mathrm{m}$ wide stripe, where the misfit dislocation density perpendicular to the direction of the stripe is about a factor of 2.4 larger than parallel to the stripe which reflects the ratio of the corresponding relaxation degrees. The asymmetry decreases with increasing stripe width and vanishes when the stripe width becomes larger than the mean TD path length in the blanket layer. Our measurements indicate that this path length is of the order of a few micrometers.

Finally, we discuss the asymmetric strains and stresses and the electronic properties expected to occur in thin Si cap stripes on relaxed SiGe stripes. Assuming for thin Si cap stripes $(<10 \mathrm{~nm})$ perfect strain transfer efficiency, we calculate the asymmetric strains along and perpendicular to the stripes, $\varepsilon_{\|, \perp}$, by using $\eta=1$ and $R=R_{\|, \perp}$ in Eq. (1). For our stripe geometry, the asymmetric biaxial stresses $\sigma_{\|, \perp}$ are related to the asymmetrical strains by

$$
\begin{aligned}
\sigma_{\|, \perp}= & {\left[\left(C_{11}+2 C_{12}\right)\left(C_{11}-C_{12}\right) / C_{11}\right]\left(\varepsilon_{\|}+\varepsilon_{\perp}\right) / 2 \pm\left(\varepsilon_{\|}\right.} \\
& \left.-\varepsilon_{\perp}\right),
\end{aligned}
$$

where $C_{11}$ and $C_{12}$ denote elastic constants of Si. According to Table I, for our narrow stripes, values as high as $\varepsilon_{\perp}$ $\approx 1 \%$ corresponding to $\sigma_{\perp} \approx 1.6 \mathrm{GPa}$ are reached.

So far, no measurements of carrier mobilities in such asymmetrically stressed thin cap layers are available. To get an idea of trends we extrapolate previous piezoelectric mea- surements on Si (Ref. 10) for tensile stresses up to about $10 \mathrm{MPa}$ to the two orders of magnitude higher stresses expected from our measurements. For our stripe geometry the anisotropic relative resistivity change in the [110] and $[-110]$ directions, $\Delta_{\|, \perp}$, is given by

$$
\left.\Delta_{\|, \perp}=\left(\Pi_{11}+\Pi_{12} \pm \Pi_{11}\right) \sigma_{\|} / 2+\Pi_{11}+\Pi_{12} \mp \Pi_{11}\right) \sigma_{\perp} / 2,
$$

where $\Pi_{i j}$ are the piezoresistivity coefficients. The high values of $\Delta_{\|, \perp}$ given in Table I (even though beyond the limits of linear piezoelectricity) indicate significant changes in the carrier mobilities. The stress induced reduction of the relative resistivity change in $n$-Si is comparable for stripe and layer structures, whereas it is substantially larger in $p$-Si for narrow stripes than for layers. For comparison, we have included in Table I values for Si layers with symmetrical biaxial strain obtained previously. ${ }^{5}$

In summary, we have investigated strain relaxation of patterned $\mathrm{SiGe}$ stripes after $\mathrm{He}^{+}$ion implantation and annealing. Asymmetric relaxation of the SiGe stripes transforms biaxial stress into strongly asymmetric stress for very narrow stripes which should yield to significant improvements of the electron and hole mobilities (Table I). The effect is explained by the limitation of the paths of threading dislocations by the stripe boundary leading to an asymmetric misfit dislocation network.

${ }^{1}$ Y. J. Mii, H. Y. Xie, E. A. Fitzgerald, Don Monroe, F. A. Thiel, B. E. Wier, and L. C. Feldman, Appl. Phys. Lett. 59, 1611 (1991).

${ }^{2}$ T. Ghani, M. Armstrong, C. Auth, M. Bost, P. Charvat, G. Glass, T. Hoffmann, K. Johnson, C. Kenyon, J. Klaus, B. McIntyre, K. Mistry, A. Murthy, J. Sandford, M. Silberstein, S. Sivakumar, P. Smith, K. Zawadzki, S. Thompson, and M. Bohr, Tech. Dig. - Int. Electron Devices Meet. 2003, 978.

${ }^{3}$ A. V.-Y. Thean, L. Prabhu, V. Varanian, M. Ramon, B.-Y Nguyen, T. White, H. Collard, Q.-H. Xie, S. Murphy, J. Cheek, S. Venkatesan, J. Mogab, C. H. Chang, Y. H. Chiu, H. C. Tuan, Y. C. See, M. S. Liang, and Y. C. Sun, Tech. Dig. - Int. Electron Devices Meet. 2005.

${ }^{4}$ Haizhou Yin, T. L. Peterson, K. D. Hobart, S. R. Shieh, T. S. Duffy, and J. C. Sturm, Appl. Phys. Lett. 87, 061922 (2005).

${ }^{5}$ D. Buca, B. Holländer, H. Trinkaus, S. Mantl, R. Carius, R. Loo, M. Caymax, and H. Schaefer, Appl. Phys. Lett. 85, 2499 (2004).

${ }^{6}$ H. Trinkaus, B. Holländer, S. Rongen, S. Mantl, H.-J. Herzog, J. Kuchenbecker, and T. Hackbarth, Appl. Phys. Lett. 76, 3552 (2000).

${ }^{7}$ D. Buca, S. Feste, B. Holländer, S. Mantl, R. Loo, M. Caymax, R. Carius, and H. Schaefer, Solid-State Electron. 50, 32 (2006).

${ }^{8}$ A. Rehman Khan, J. Stangl, G. Bauer, D. Buca, B. Holländer, H. Trinkaus, S. Mantl, R. Loo, and M. Caymax, Semicond. Sci. Technol. 22, 212 (2007); H. Trinkaus, D. Buca, B. Hollander, and S. Mantl, Conference Digest of the Third International Silicon Germanium Technology and Devices Meeting, Princeton, NJ, 2006, p. 120.

${ }^{9}$ B. Holländer, S. Lenk, S. Mantl, H. Trinkaus, D. Kirch, M. Luysberg, T. Hackbarth, H.-J. Herzog, and P. Fichtner, Nucl. Instrum. Methods Phys. Res. B 175-177, 357 (2001).

${ }^{10}$ Charles S. Smith, Phys. Rev. 94, 42 (1954). 